\title{
Transition of a partially yielded Casson fluid from circular to helical flow
}

\author{
F. M. Alharbi ${ }^{1} \quad$ J. J. Shepherd ${ }^{2} \quad$ A. J. Stacey ${ }^{3}$
}

(Received 28 February 2015; revised 19 January 2016)

\begin{abstract}
In the Casson model, a fluid behaves as a solid for low stress but yields to flow as a viscoplastic fluid as the stress increases beyond a yield stress. The Casson model is often used to model the flow of blood or the flows occurring in food processing. We analyze the flow of a Casson fluid between infinitely long coaxial cylinders, with the inner cylinder rotating and the outer cylinder stationary, so that only the fluid adjacent to the inner cylinder yields. Simultaneously, axial flow arising from a small axial pressure gradient causes the flow to transform from a circular to a helical flow. Such flow is relevant to a number of applications, particularly rheometry. A perturbation analysis based on the pressure gradient provides explicit approximations for the fluid velocity profiles, as well as the change in location of the
\end{abstract}

http://journal.austms.org.au/ojs/index.php/ANZIAMJ/article/view/9364 gives this article, (c) Austral. Mathematical Soc. 2016. Published January 31, 2016, as part of the Proceedings of the 17th Biennial Computational Techniques and Applications Conference. ISSN 1446-8735. (Print two pages per sheet of paper.) Copies of this article must not be made otherwise available on the internet; instead link directly to this URL for this article. 
solid-fluid boundary. These approximations show the dependence of flow quantities on a range of fluid parameters, not just for specific parameter values, as occurs when numerical calculations are used.

\section{Contents}

1 Introduction

C297

2 Governing equations

C299

3 Dimensionless formulation

C300

4 Perturbation analysis

C302

4.1 Leading order terms . . . . . . . . . . . . . .

C303

4.2 Higher order terms . . . . . . . . . . . . . .

C304

5 Discussion

C306

References

C309

\section{Introduction}

Yield stress fluids behave like solids for lower values of the total fluid stress, but when this stress exceeds a given value, the yield stress, the solid yields to flow as a (generally, non-Newtonian) fluid. Such fluids are encountered in many medical and industrial applications $[1,4]$. Flows of such fluids may contain solid and fluid regions, separated by yield boundaries or freeze boundaries, where the stress has the yield value.

The simplest example of a yield stress fluid is the Bingham fluid [3, e.g.], which flows as a Newtonian fluid beyond yield and was used to model the flow of paints [2]. Another example is the Casson fluid, which flows as a 
shear-thinning non-Newtonian fluid beyond yield and was used to model the flow of blood [4]. There are many other examples of yield stress fluids displaying a wide variety of characteristics, as discussed by Bird et al. [3, Chap. 4] and Tanner [11, Chap. 1].

The geometry of the flow of a yield stress fluid determines its flow characteristics; in particular, the existence and location of any yield zones and yield boundaries. Among many geometries, one of interest occurs in fluid flows between two infinitely long coaxial cylinders. With the steady rotation of one of the cylinders as well as a constant axial pressure gradient applied to the fluid in the intercylindrical gap, helical flow results, with the particle paths forming helices.

While exact solutions of the equations of motion for helical flow of Newtonian fluids are readily found [8, Chap. 4], exact solutions for the non-linear constitutive equations describing non-Newtonian fluids are impossible and numerical solution procedures must be employed. Coleman and Noll [6] gave a general solution for helical flow of a general non-Newtonian fluid, but their very general solution is of limited applicability and says nothing about yield stress fluids with yield zones in the flow. In certain helical flows, analytical (as distinct from numerical) approximations for the flow field of non-Newtonian fluids can be obtained. In particular, when a small parameter is identified in the equations, perturbation methods provide very satisfactory approximations [5, 9, 7, e.g.].

In this article we examine the helical flow of a Casson fluid flowing between two coaxial cylinders, with the inner cylinder rotating and the outer cylinder held stationary, applying a small axial pressure gradient. The rotational shearing motion is assumed to provide enough stress for the fluid next to the inner cylinder to yield, producing a fluid region adjacent to the inner cylinder and a yield boundary in the intercylindrical gap. We apply a perturbation method based on the axial pressure gradient to obtain explicit expressions for the fluid velocity field and the location of the yield boundary in the flow. This approach parallels that of Shepherd et al. [10] for the yielded helical flow 
of a Bingham fluid.

\section{Governing equations}

We consider the steady helical flow of an incompressible viscous fluid between infinitely long coaxial cylinders, the inner cylinder with radius $R_{i}$ and the outer cylinder with radius $R_{0}$. The region between the cylinders is described in cylindrical polar coordinates $(r, \theta, z)$, where $R_{i} \leqslant r \leqslant R_{o}, 0 \leqslant \theta \leqslant 2 \pi$, $-\infty \leqslant z \leqslant \infty$. The inner cylinder has a constant angular velocity $\Omega>0$, while the outer cylinder is held stationary. It is assumed that $\Omega$ is such that only the fluid closest to the inner cylinder will yield, resulting in a liquid-solid boundary at $r=\bar{R}<R_{0}$. In addition, we impose a small axial pressure gradient $A<0$, which is such that a liquid-solid boundary is retained.

The axes of the cylinders are chosen to lie on the $z$-axis, with the axial component of the flow in the positive $z$-axis direction. Like Shepherd et al. [10] who dealt with a Bingham fluid, the velocity field is $\left(\mathfrak{u}_{r}, \mathfrak{u}_{\theta}, \mathfrak{u}_{z}\right)=(0, r W(r), V(r))$ for appropriate functions $V(r), W(r)$ and the field satisfies conservation of mass. The momentum equations then yield differential equations for $V(r)$ and $W(r)$ within the fluid region,

$$
\begin{aligned}
& \left(\sqrt{\frac{T_{o}}{K(r)}}+\sqrt{H_{o}}\right)^{2} V^{\prime}(r)=\frac{A}{r}\left(r^{2}-\gamma^{2}\right), \\
& \left(\sqrt{\frac{T_{o}}{K(r)}}+\sqrt{H_{o}}\right)^{2} W^{\prime}(r)=\frac{-M}{2 \pi r^{3}},
\end{aligned}
$$

where the two positive constants $T_{o}$ and $H_{o}$ are the yield stress and viscosity, respectively, which are given properties of the fluid, $\gamma$ is an integration constant to be determined, $M>0$ is interpreted as the moment per unit length exerted on the inner cylinder and $A$ is the pressure gradient. Both 
$M$ and $A$ are given and drive the helical flow. The local rate of shearing is written in terms of $V^{\prime}$ and $W^{\prime}$ as $K(r)=\sqrt{\left[\left(r W^{\prime}\right)^{2}+V^{\prime 2}\right] / 2}$.

Dividing the first equation of (1) by the second gives

$$
V^{\prime}(r)=-2 \pi A r^{2}\left(r^{2}-\gamma^{2}\right) W^{\prime}(r) / M
$$

The non-slip conditions on the inner cylinder and at the cylindrical freeze boundary, plus the condition that $\mathrm{K}=0$ at the freeze boundary provide the boundary conditions

$$
\mathrm{V}\left(\mathrm{R}_{\mathrm{i}}\right)=\mathrm{V}(\overline{\mathrm{R}})=\mathrm{V}^{\prime}(\overline{\mathrm{R}})=0, \quad \mathrm{~W}\left(\mathrm{R}_{\mathrm{i}}\right)=\Omega, \quad \mathrm{W}(\overline{\mathrm{R}})=\mathrm{W}^{\prime}(\overline{\mathrm{R}})=0 .
$$

The second equation of (1) implies that $W^{\prime}(r)<0$ over the region $R_{i}<r<\bar{R}$, hence $\left|W^{\prime}\right|=-W^{\prime}$ and we write the local rate of shearing as

$$
K=-\frac{r W^{\prime} \phi(r, A, \gamma)}{\sqrt{2}}, \quad \text { for } \phi(r, A, \gamma)=\sqrt{1+\left(\frac{2 \pi A}{M}\right)^{2} r^{2}\left(r^{2}-\gamma^{2}\right)^{2}}
$$

Substituting (4) into the second equation of (1) yields a quadratic in $W^{\prime}$ which when solved subject to $W^{\prime}<0$ gives

$$
W^{\prime}=-\frac{\sqrt{2} T_{o}}{H_{o} r}\left(\frac{1}{\sqrt{\phi(r, A, \gamma)}}-\sqrt{\frac{M}{2 \sqrt{2} \pi T_{o} r^{2}}}\right)^{2} .
$$

\section{Dimensionless formulation}

Define characteristic radial distance $R$, dimensionless radial variable $s$ and dimensionless constants $s_{i}, s_{0}, \bar{s}$ and $\sigma$ by

$$
\begin{aligned}
& R=\left(R_{i}+R_{o}\right) / 2, \quad r=R s, \quad R_{i}=R s_{i}, \\
& R_{o}=R s_{o}, \quad \bar{R}=R \bar{s}, \quad \gamma=R \sigma
\end{aligned}
$$


and dimensionless forms of $\mathrm{V}$ and $\mathrm{W}$ by

$$
v(s)=\Upsilon V(R s) / R, \quad w(s)=\Upsilon W(R s), \quad \text { where } \Upsilon=2 \pi \mathrm{H}_{0} R^{2} / M .
$$

The dimensionless forms of the equations of motion (5) and (2) are

$$
w^{\prime}(s)=-\frac{1}{s^{3}}\left(s \sqrt{\frac{\tau_{o}}{\psi}}-1\right)^{2}, \quad v^{\prime}(s)=a s^{2}\left(s^{2}-\sigma^{2}\right) w^{\prime}(s)
$$

respectively, where

$$
\psi=\sqrt{1+(s a)^{2}\left(s^{2}-\sigma^{2}\right)^{2}}, \quad a=-\frac{2 \pi A R^{3}}{M}, \quad \tau_{o}=\frac{2 \sqrt{2} \pi T_{0} R^{2}}{M} .
$$

The non-slip boundary conditions and freeze boundary conditions (3) become, respectively,

$$
v\left(s_{i}\right)=0, \quad w\left(s_{i}\right)=\omega=2 \pi \mathrm{H}_{0} R^{2} \Omega / M,
$$

and

$$
v(\bar{s})=0, \quad w(\bar{s})=0, \quad w^{\prime}(\bar{s})=0 .
$$

The unknown quantities in problem (8)-(11) are the functions $\boldsymbol{w}(\mathrm{s})$ and $\boldsymbol{v}(\mathrm{s})$, and the constants $\sigma, \bar{s}$ and $\omega$. We view a as a known quantity.

In principle, the first equation of (8) may be integrated, and using the second equation of (11) we can solve for the constant of integration in terms of the unknown $\sigma$. If we then apply the last equation of (10), then we get an equation involving the three unknowns $\sigma, \bar{s}$ and $\omega$. Similarly, we may integrate the second equation of (8) and apply the first equations of both (10) and (11) to get a second equation relating $\sigma, \bar{s}$ and $\omega$. Finally, applying the last equation of (11) to the first equation of (8) gives an equation in the two unknowns $\sigma$ and $\bar{s}$. Solving this system of three equations would provide a complete solution. 
Equations (8)-(11) are highly nonlinear and in most cases must be solved numerically. In what follows we consider the special case where the flow is dominated by rotation and has a small axial flow component. The small axial flow, being caused by a small pressure gradient $A$, corresponds to a small $a$ in the nondimensional formulation. This small parameter $a$ is used as a perturbation parameter to find approximate analytic expressions for unknown quantities $w(s), v(s), \sigma$ and $\bar{s}$, and from these we calculate $\omega$.

\section{Perturbation analysis}

We use perturbation expansions of $\mathcal{w}(\mathrm{s}), v(\mathrm{~s}), \sigma$ and $\bar{s}$ in the small parameter $a>0$. Then we simplify the calculations that follow by applying an argument analogous to that applied by Shepherd et al. [10]. Under this argument, changing the sign of a corresponds to changing the sign of $A$ and physical arguments lead us to assert that $\bar{s}, \sigma$ and $w(s)$ are even functions of $a$, while $v(s)$ is an odd function of $a$. Thus we propose the expansions

$$
\begin{aligned}
\bar{s} & \sim \bar{s}_{0}+\bar{s}_{2} a^{2}+\bar{s}_{4} a^{4}+\cdots \\
\sigma & \sim \sigma_{0}+\sigma_{2} a^{2}+\sigma_{4} a^{4}+\cdots \\
v(s) & \sim v_{1}(s) a+v_{3}(s) a^{3}+v_{5}(s) a^{5}+\cdots \\
w(s) & \sim w_{0}(s)+w_{2}(s) a^{2}+w_{4}(s) a^{4}+\cdots .
\end{aligned}
$$

Substituting (13), (14) and (15) into (8) and equating like powers of a produces a sequence of differential equations for the first few terms of $v(s)$ and $w(s)$ :

$$
\begin{aligned}
w_{0}^{\prime}(s) & =-\frac{1}{s^{3}}\left(s \sqrt{\tau_{0}}-1\right)^{2}, \\
w_{2}^{\prime}(s) & =\frac{1}{2} \sqrt{\tau_{0}}\left(s \sqrt{\tau_{0}}-1\right)\left(s^{2}-\sigma_{0}^{2}\right)^{2} \\
v_{1}^{\prime}(s) & =s^{2}\left(s^{2}-\sigma_{0}^{2}\right) w_{0}^{\prime}(s), \\
v_{3}^{\prime}(s) & =s^{2}\left(s^{2}-\sigma_{0}^{2}\right) w_{2}^{\prime}(s)-2 \sigma_{0} \sigma_{2} s^{2} w_{0}^{\prime}(s) .
\end{aligned}
$$


Applying the last equation of (11) to (15) gives

$$
w^{\prime}(\bar{s})=w_{0}^{\prime}(\bar{s})+w_{2}^{\prime}(\bar{s}) a^{2}+w_{4}^{\prime}(\bar{s}) a^{4}+\cdots=0 .
$$

Using (12), expanding each of the $\boldsymbol{w}_{\mathfrak{i}}^{\prime}(\overline{\boldsymbol{s}})$ about $\overline{\boldsymbol{s}}_{0}$, and equating coefficients of powers of a gives

$$
w_{0}^{\prime}\left(\bar{s}_{0}\right)=0, \quad w_{0}^{\prime \prime}\left(\bar{s}_{0}\right) \bar{s}_{2}+w_{2}^{\prime}\left(\bar{s}_{0}\right)=0 .
$$

Similarly, applying (12) and (15) to the second equation of (11), and with the first equation of (20), gives

$$
w_{0}\left(\bar{s}_{0}\right)=0, \quad w_{2}\left(\bar{s}_{0}\right)=0 .
$$

In a like manner the first equations of (10) and (11) produce, on applying the first equations of both (21) and (18),

$$
\begin{aligned}
& v_{1}\left(\bar{s}_{0}\right)=v_{1}\left(s_{i}\right)=0, \\
& v_{3}\left(\bar{s}_{0}\right)=v_{3}\left(s_{i}\right)=0 .
\end{aligned}
$$

\subsection{Leading order terms}

Here we consider the leading order terms of (12)-(13). This corresponds to purely annular flow in the inter-cylinder gap.

From (20) and (16),

$$
\overline{\mathrm{s}}_{0}=\frac{1}{\sqrt{\tau_{\mathrm{o}}}},
$$

determining $\bar{s}_{0}$. This also makes $w_{2}^{\prime}(s)<0$ on $s_{i}<s<\bar{s}_{0}$.

Integrating (16) subject to the first boundary condition of (21) gives

$$
w_{0}(s)=\tau_{0} \log \left(\frac{\bar{s}_{0}}{s}\right)+2 \sqrt{\tau_{0}}\left(\frac{1}{\bar{s}_{0}}-\frac{1}{s}\right)+\frac{1}{2}\left(\frac{1}{s^{2}}-\frac{1}{\bar{s}_{0}^{2}}\right) .
$$


Finally, integrating (18) subject to the boundary conditions (22) gives

$$
\sigma_{0}^{2}=\frac{\frac{\tau_{0}}{4}\left(\bar{s}_{0}^{4}-s_{i}^{4}\right)-\frac{2 \sqrt{\tau_{0}}}{3}\left(\bar{s}_{0}^{3}-s_{i}^{3}\right)+\frac{1}{2}\left(\bar{s}_{0}^{2}-s_{i}^{2}\right)}{\frac{\tau_{0}}{2}\left(\bar{s}_{0}^{2}-s_{i}^{2}\right)-2 \sqrt{\tau_{0}}\left(\bar{s}_{0}-s_{i}\right)+\log \left(\bar{s}_{0} / s_{i}\right)},
$$

determining $\sigma_{0}$. Since $v(s)$ is assumed continuous and it must satisfy the boundary conditions (10) and (11), $v^{\prime}(\mathrm{s})$ must change sign on the domain $s_{i} \leqslant s \leqslant \bar{s}_{0}$. Since $w^{\prime}(s)$ does not change sign on $s_{i} \leqslant s \leqslant \bar{s}_{0}$, the sign change of $v^{\prime}(\mathrm{s})$ is only possible if $\sigma^{2}>0$.

\subsection{Higher order terms}

We now consider $\mathcal{O}\left(\mathbf{a}^{2}\right)$ terms of (12)-(13). These terms give corrections to the $\mathcal{O}(1)$ terms.

Rearranging the second equation of (20) and using (16) and (17) gives

$$
\bar{s}_{2}=\bar{s}_{0}^{4} \sqrt{\tau_{\mathrm{o}}}\left(\bar{s}_{0}^{2}-\sigma_{0}^{2}\right)^{2} / 4 .
$$

The coefficient $\sigma_{2}$ is determined by integrating (19) subject to boundary conditions (23):

$$
\sigma_{2}=\frac{\int_{s_{i}}^{\bar{s}_{0}} s^{2}\left(s^{2}-\sigma_{0}^{2}\right) w_{2}^{\prime}(s) d s}{2 \sigma_{0} \int_{s_{i}}^{\bar{s}_{0}} s^{2} w_{0}^{\prime}(s) d s} .
$$

Next we find expressions for $w_{2}(s), v_{1}(s)$ and $v_{3}(s)$. To find $w_{2}(s)$ we integrate (17) and apply the second equation of (21), to find $v_{1}(s)$ we integrate (18) and apply the second equation of (22), and finally, to find $v_{3}(s)$ we 
integrate (19) and apply the second equation of (23):

$$
\begin{aligned}
w_{2}(s)= & H(s)-H\left(\bar{s}_{0}\right), \\
v_{1}(s)= & \frac{\tau_{0}}{4}\left(s^{4}-s_{i}^{4}\right)+\frac{2 \sqrt{\tau_{0}}}{3}\left(s^{3}-s_{i}^{3}\right)+\frac{\sigma_{0}^{2} \tau_{0}}{2}\left(s^{2}-s_{i}^{2}\right) \\
& -\frac{1}{2}\left(s^{2}-s_{i}^{2}\right)-2 \sigma_{0}^{2} \sqrt{\tau_{0}}\left(s-s_{i}\right)+\sigma_{0}^{2} \log \left(\frac{s}{s_{i}}\right), \\
v_{3}(s)= & G(s)-G\left(s_{i}\right),
\end{aligned}
$$

where

$$
\begin{aligned}
H(s)= & \frac{\sqrt{\tau_{\mathrm{o}}}}{2}\left(\frac{1}{6} \sqrt{\tau_{\mathrm{o}}} s^{6}-\frac{1}{5} s^{5}-\frac{1}{2} \sigma_{0}^{2} \sqrt{\tau_{\mathrm{o}}} s^{4}+\frac{2}{3} \sigma_{0}^{2} s^{3}+\frac{1}{2} \sigma_{0}^{4} \sqrt{\tau_{\mathrm{o}}} s^{2}-\sigma_{0}^{4} s\right) \\
\mathrm{G}(\mathrm{s})= & \frac{\tau_{\mathrm{o}}}{20} s^{10}-\frac{\sqrt{\tau_{\mathrm{o}}}}{18} s^{9}-\frac{3 \tau_{\mathrm{o}} \sigma_{0}^{2}}{16} s^{8}+\frac{3 \sigma_{0}^{2} \sqrt{\tau_{\mathrm{o}}}}{14} s^{7}+\frac{\tau_{\mathrm{o}} \sigma_{0}^{4}}{4} s^{6}-\frac{\tau_{\mathrm{o}} \sigma_{0}^{6}}{8} s^{4} \\
& -\frac{3 \sigma_{0}^{4} \sqrt{\tau_{\mathrm{o}}}}{10} s^{5}+\frac{\sigma_{0}^{6} \sqrt{\tau_{\mathrm{o}}}}{6} s^{3}+\sigma_{0} \sigma_{2}\left(\tau_{\mathrm{o}} s^{2}-4 \sqrt{\tau_{\mathrm{o}}} s+2 \log s\right)
\end{aligned}
$$

Similarly, applying the boundary condition given by the second equation of (10) to the perturbation expansion for $w$ given by (15) gives an expression for the angular velocity of the inner cylinder:

$$
\omega=\omega_{0}+\omega_{2} a^{2}+\mathcal{O}\left(a^{4}\right),
$$

where

$$
\begin{aligned}
\omega_{0}= & \frac{1}{2 s_{i}^{2} \bar{s}_{0}^{2}}\left(4 \sqrt{\tau_{0}}\left(s_{i}^{2} \bar{s}_{0}-s_{i} \bar{s}_{0}^{2}\right)+2 \tau_{0} s_{i}^{2} \bar{s}_{0}^{2} \log \left(\frac{\bar{s}_{0}}{s_{i}}\right)+\bar{s}_{0}^{2}-s_{i}^{2}\right), \\
\omega_{2}= & -\frac{\tau_{0}}{2}\left(\frac{1}{6}\left(\bar{s}_{0}^{6}-s_{i}^{6}\right)-\frac{\sigma_{0}^{2}}{2}\left(\bar{s}_{0}^{4}-s_{i}^{4}\right)+\frac{\sigma_{0}^{4}}{2}\left(\bar{s}_{0}^{2}-s_{i}^{2}\right)\right) \\
& -\frac{\sqrt{\tau_{0}}}{2}\left(\frac{1}{5}\left(\bar{s}_{0}^{5}-s_{i}^{5}\right)+\frac{2 \sigma_{0}^{2}}{3}\left(\bar{s}_{0}^{3}-s_{i}^{3}\right)-\sigma_{0}^{4}\left(\bar{s}_{0}-s_{i}\right)\right) .
\end{aligned}
$$




\section{Discussion}

The expansions (12), (13), (14) and (15) are explicit, readily computed approximate expressions for the fluid velocity components $v(s)$ and $\mathcal{w}(\mathrm{s})$, and the constants $\bar{s}$ and $\sigma$. We expect these to be accurate for suitably small positive values of the dimensionless parameter $a$. Note that $a=0$ corresponds to annular flow (no axial flow) with $v(s)=0$ and the leading order terms (24), (25), (26) and (34) describe the flow. The velocity swo $(s)$ is transverse and $\bar{s}_{0}(24)$, which depends only on $\tau_{0}$, locates the yield boundary. The rate of rotation $\omega_{0}$ is dependent on $\tau_{0}$ and the cylinder geometry through (34). The Reiner-Riwlin relationship (33) links all these parameters to $\Omega$ and a measurement of $\Omega$ allows estimation of the fluid parameters $T_{0}$ and $H_{0}$.

For $\mathbf{a}>0,(14)$ and (15) describe the emerging helical flow field with both transverse and axial components $v(s)$ and $\boldsymbol{w}(\mathbf{s})$, while (12) and (13), respectively, show variations in the yield boundary and axial velocity peak due to the axial flow. From (27), $\bar{s}_{2}>0$, that is, the yield boundary is shifted outward, quantifying what is predicted qualitatively on physical grounds. Similarly, since (17) gives $w_{2}^{\prime}(s)<0$ on $s_{i}<s<\bar{s}_{0}$, and from the second equation of $(21), w_{2}\left(\bar{s}_{0}\right)=0$, we deduce that $\omega_{2}=w_{2}\left(s_{i}\right)>0$. Thus, the axial flow has the effect of increasing the angular speed of the fluid at the inner cylinder.

We solved the helical flow problem numerically. Numerical solutions for $w(s)$ and $v(s)$ are possible once $\sigma, \bar{s}$ and $\omega$ are known after numerically integrating (8) using appropriate boundary conditions.

To find values for $\sigma$ and $\bar{s}$ we used the two constraint equations $w^{\prime}(\bar{s})=0$ and $\int_{s_{i}}^{\bar{s}} v^{\prime}(s) d s=0$. These equations have the form $f_{i}(\sigma, \bar{s})=0$ for $i=1,2$. The Matlab function fminsearch was used to find the point $(\sigma, \bar{s})$ which minimizes the maximum of the two functions $\left|f_{i}(\sigma, \bar{s})\right|$. Typical maximum values of $\left|f_{\mathfrak{i}}(\sigma, \bar{s})\right|$ are of the order of $10^{-17}$. This minimization problem is non-trivial; a good solution is only found if the initial guess is close to the exact solution. For this reason, for the initial estimate of $(\sigma, \bar{s})$ we used our 
Figure 1: Velocity profile for $w(s)$ where $\tau_{\mathrm{o}}=0.7583, s_{i}=0.1250, \bar{s}=1.1556$ and the numeric freeze radius $\bar{s}=1.1558$.

$\mathrm{a}=0.1$

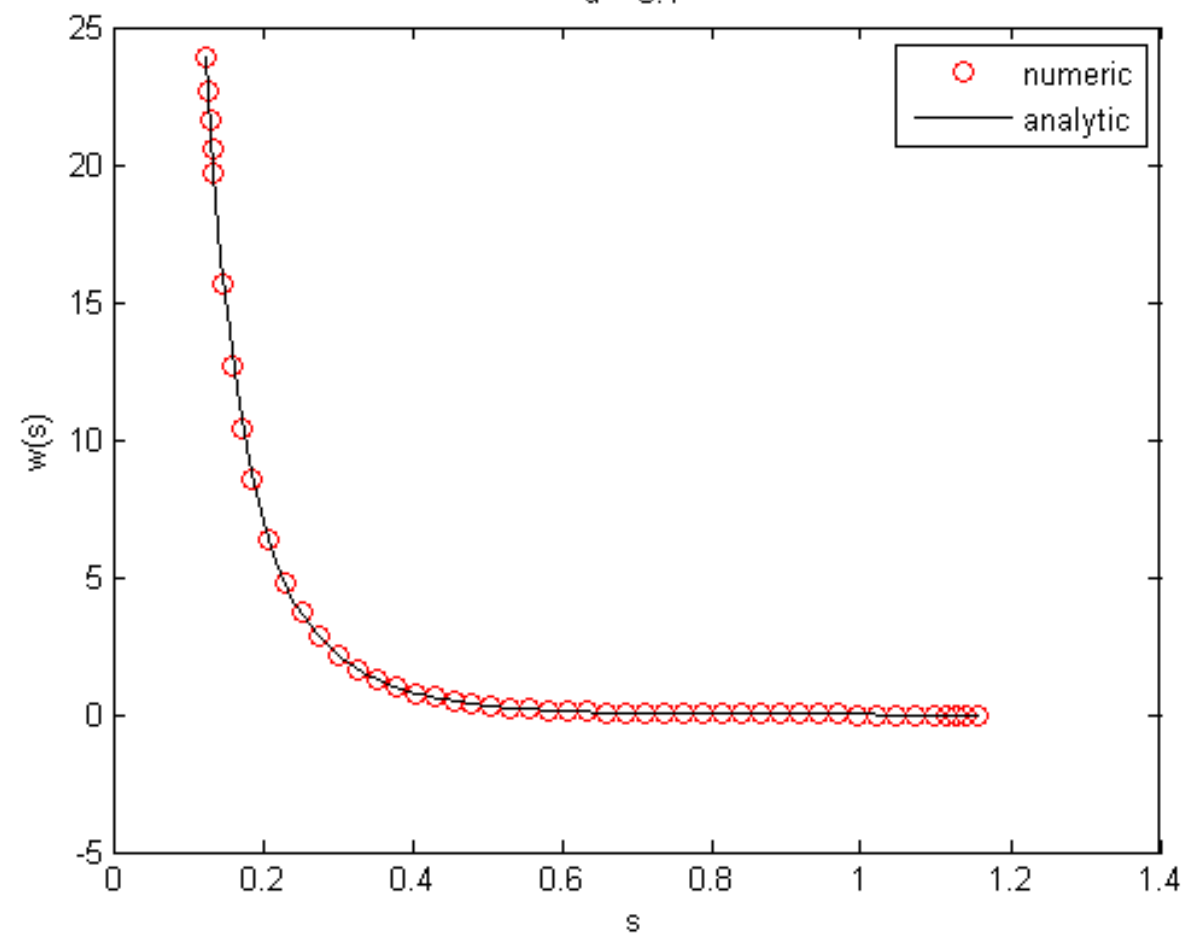

analytic approximation. It is also important that the numerical integration over $v^{\prime}(s)$ in the second constraint is sufficiently accurate. Once $\sigma$ and $\bar{s}$ are known, the angular velocity is calculated from $\omega=-\int_{s_{i}}^{\bar{s}} w^{\prime}(s) d s$.

Figures 1 and 2 display velocity components $v(s)$ and $\mathcal{w}(\mathrm{s})$ as given by the first two terms of (14) and (15), across the intercylindrical gap for appropriate choices of the fluid parameters, cylinders geometry and for small a. The figures compare analytic and numerical results and show very good agreement. Table 1 reinforces the agreement, giving the errors of the numerical results 
Figure 2: Velocity profile for $v(\mathrm{~s})$ where $\tau_{\mathrm{o}}=0.7583, s_{\mathrm{i}}=0.1250, \overline{\mathrm{s}}=1.1556$ and the numeric freeze radius $\bar{s}=1.1558$.

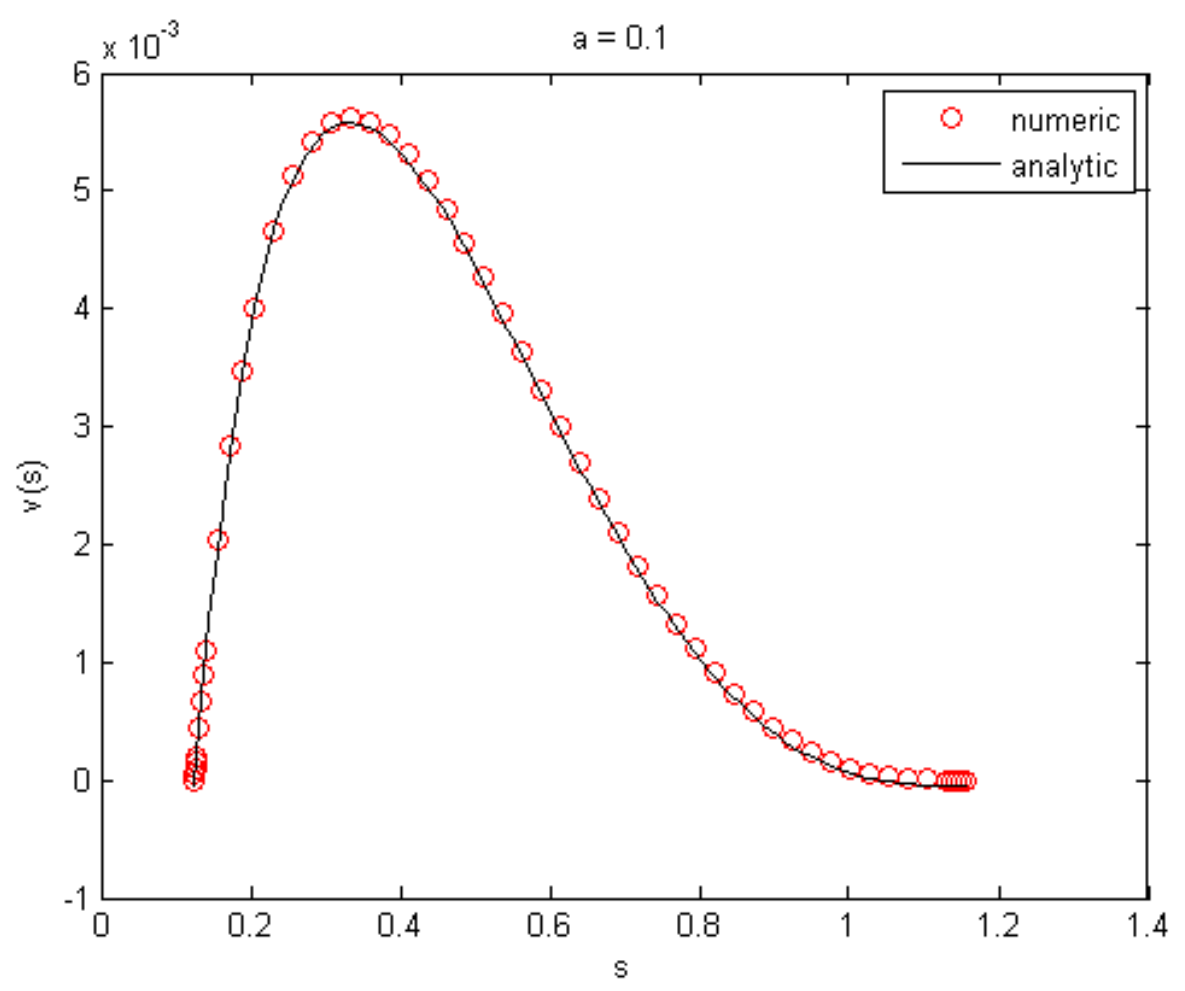

compared to the analytical results for parameters $\sigma, \bar{s}$ and $\omega$ for several small values of $a$.

Acknowledgements F. M. Alharbi thanks the University of Tabuk for financial support for his PhD studies at RMIT University. 
Table 1: The absolute value of the difference between the numeric and analytic solution for the flow parameters for different a values.

\begin{tabular}{c|ccc} 
& \multicolumn{3}{|c}{ Error } \\
& $a=0.1$ & $a=0.01$ & $a=0.001$ \\
\hline$\sigma$ & $0.1728 \times 10^{-7}$ & $0.1698 \times 10^{-11}$ & $0.1720 \times 10^{-15}$ \\
$\bar{s}$ & $0.1324 \times 10^{-5}$ & $0.1279 \times 10^{-9}$ & $0.1063 \times 10^{-11}$ \\
$\omega$ & $0.3866 \times 10^{-5}$ & $0.3939 \times 10^{-6}$ & $0.1193 \times 10^{-8}$
\end{tabular}

\section{References}

[1] E. O. Afoakwa, A. Paterson and M. Fowler. Factors influencing rheological and textural qualities in chocolate - a review. Trends Food Sci. Tech., 18(6):290-298, 2007. doi:10.1016/j.tifs.2007.02.002 C297

[2] E. C. Bingham. Fluidity and plasticity. McGraw-Hill, New York 1922. $\mathrm{C} 297$

[3] R. B. Bird, R. C. Armstrong and O. Hassager. Dynamics of Polymeric Liquids, Volume 1, Fluid Mechanics. John Wiley and Sons New York, 1987. http://au.wiley.com/WileyCDA/WileyTitle/ productCd-047180245X.html C297, C298

[4] G. W. Scott Blair. An equation for the flow of blood, plasma and serum through glass capillaries. Nature, 183:613-614, 1959. doi:10.1038/183613a0 C297, C298

[5] C. Chiera, H. J. Connell and J. J. Shepherd. A perturbation approach to the analysis of a Casson model fluid, in Proceedings of the Second Biennial Australian Engineering Mathematics Conference: 1996; Engineering Mathematics; Research, Education and Industry Linkage. Institution of Engineers, Australia, pp. 315, 1996. http://search. informit.com.au/documentSummary; dn= 718952602745151 ; res=IELENG C298 
[6] B. D. Coleman and W. Noll. Helical flow of general fluids, J. Appl. Phys., 30:1508-1512, 1959. doi:10.1063/1.1734990 C298

[7] M. T. Farrugia, J. J. Shepherd and A. J. Stacey. A perturbation analysis of the flow of a Powell-Eyring fluid between coaxial cylinders, CTAC2010, ANZIAM J., 52:C257-C270, 2011. http://journal. austms.org.au/ojs/index.php/ANZIAMJ/article/view/3748 C298

[8] W. E. Langlois. Slow viscous flow. Macmillan New York, 1964. doi:10.1007/978-3-319-03835-3 C298

[9] J. J. Shepherd, C. Chiera and H. J. Connell. Perturbation analysis of the helical flow of non-Newtonian fluids with application to a recirculating coaxial cylinder rheometer, Math. Comput. Model., 18(10):131-140, 1993. doi:10.1016/0895-7177(93)90222-K C298

[10] J. J. Shepherd, A. J. Stacey, and A. A. Khan, Helical flow arising from the yielded annular flow of a Bingham fluid, Appl. Math. Model., 38(23):5382-5391, 2014. doi:10.1016/j.apm.2014.04.031 C298, C299, C302

[11] R. I. Tanner. Engineering Rheology, Oxford University Press, New York, 1988. https://global.oup.com/academic/product/ engineering-rheology-9780198564737?cc=au\&lang=en\& C298

\section{Author addresses}

1. F. M. Alharbi, School of Mathematical and Geospatial Sciences, RMIT University, Melbourne, Victoria 3000, Australia.

2. J. J. Shepherd, School of Mathematical and Geospatial Sciences, RMIT University, Melbourne, Victoria 3000, Australia. mailto: john. shepherd@rmit.edu.au 
3. A. J. Stacey, School of Mathematical and Geospatial Sciences, RMIT University, Melbourne, Victoria 3000, Australia. 